\title{
Monstruos de Mujica Lainez: clase, cuerpo y medida*
}

\author{
Marcos Zangrandi ${ }^{* *}$
}

\begin{abstract}
Resumen
Este trabajo examina la categoría de monstruosidad en varias obras del narrador argentino Manuel Mujica Lainez. Por un lado indaga en estas configuraciones ligadas directamente a la visión erotizada de los cuerpos. Contiguamente, se analiza el cruce entre monstruos y estatuas, figuras habituales de la literatura de Mujica, que señalan la gradación de belleza y fealdad, y que llenan de presunción o atormentan a los distintos personajes. Finalmente este artículo indaga un aspecto original del escritor: la insistencia de proponer la monstruosidad como forma válida de diferencia social que caracteriza a las clases altas.
\end{abstract}

Palabras clave: literatura argentina- monstruosidad- clase social- género.

\section{Monsters of Mujica Lainez: class, body, and measure}

\begin{abstract}
The paper examines the category of monstrosity in several works of Manuel Mujica Lainez. Firstly explores the appearance of extraordinary creatures, result from the mixture of several animal species whose emergence is directly linked to the view of sex. Then, the paper examines the intersection between monsters and statues, usual images of Mujica literature, which show gradation of beauty and ugliness, and cause equally torment or vanity to the characters. Finally, the essay explores a Mujica's exceptional point of view: he insists to reveal the monstrosity as a valid form of aristocracy.
\end{abstract}

Key words: Argentine literature- monstrosity- social class- gender.

Recibido: 19-08-2013 Aceptado: 11-11-2013

* Este artículo se desprende de una investigación doctoral dedicada a las narrativas de la familia durante las décadas de 1950 y 1960 en Argentina, financiada por el Consejo Nacional de Investigaciones Científicas y Técnicas (CONICET).

** Argentino, Magíster en Comunicación y Cultura de la Universidad de Buenos Aires, Instituto de Literatura Hispanoamericana, Universidad de Buenos Aires, Consejo Nacional de Investigaciones Científicas y Técnicas (CONICET), Buenos Aires, Argentina, marcoszangrandi@gmail.com 
Según la caracterización que se desprende de la tradición gótica, la representación de las monstruosidades en la producción cultural y los imaginarios colectivos ponen de manifiesto los límites de las sociedades que los generan. Como corporalidad defectuosa, duplicada, fragmentada, híbrida o gigantesca, el monstruo causa pavor porque encarna las amenazas y revela las fronteras políticas y culturales (Cohen, 1998). La ensayista Susan Stewart (2007) apunta, al respecto, que la etimología del término es clara -proviene de los vocablos moneo, advertir y monstro, mostrar-, ya que el monstruo es un señalero de aquella línea que no se debe cruzar. Alberto Manguel interpreta, bajo esta misma perspectiva, que el monstruo es un marca que previene: "guárdate de adentrarte en estas tierras" (2008, 15). De este modo, en el cuerpo monstruoso pueden leerse aquellas señales con que cada sociedad establece los estándares de normalidad, inclusión y marginación, así como sus contradicciones y los horizontes que separan lo posible de lo vedado.

El narrador argentino Manuel Mujica Lainez (1910-1984) debate la construcción de la monstruosidad como borde sociocultural ${ }^{1}$. En primer lugar, sitúa al cuerpo extraño e inclasificable como objeto del deseo; efectivamente, en algunas de sus narraciones ("El retrato amarillo" [1956], El viaje de los siete demonios [1974], Sergio [1976] y Los cisnes [1977]), el monstruo aparece ficcionalizado como visión repulsiva, pero lo es de tal manera quelos observadores, antes de reafirmar una posición aséptica, se ven implicados y movilizados hacia este ser que está fuera de los estándares y de las expectativas. En otros textos, en cambio, puede advertirse repetidamente la asunción del punto de vista de la criatura anómala para evidenciar las posibilidades de diferenciación social que otorga la monstruosidad, como se puede observar en las novelas Invitados en 'El Paraíso' (1957), Bomarzo (1962) y Cecil (1975). En el recorrido de estas configuraciones se advierte, asimismo, el diálogo de los textos (y de una fracción de la cultura) con una coyuntura de fuerte discusión política y cultural y de alteración de la lógica social a lo largo de la primera mitad del siglo $\mathrm{XX}^{2}$.

1 Sobre la figura y la trayectoria de Manuel Mujica Lainez ver los estudios de Jorge Cruz (1997) y Oscar Hermes Villordo (1991). Gastón Gallo (2004) realizó, asimismo, un estudio general sobre la obra de Mujica Lainez dentro del panorama de la literatura argentina del siglo XX. Dos textos introductorios de Alejandra Laera $(2005,2007)$ abren puntos de vista innovadores sobre la narrativa del escritor argentino, por ejemplo las que se vinculan con la perspectiva de género, prácticamente inexplorada hasta entonces.

2 Sobre esas transformaciones y sobre todo en relación con el ingreso del peronismo en la vida argentina ver las referencias históricas en los tomos dirigidos por Juan Carlos Torre (2002) y Daniel James (2007). Respecto de los debates culturales durante los años 1950 y 1960 ver los estudios de Oscar Terán (1991) y Claudia Gilman (2003). 


\section{Monstruosidad, deseo y nostalgia}

Una línea ficcional de Mujica Lainez redunda sobre la monstruosidad enlazada al sexo, escena que causa al mismo tiempo pavor y deseo. Esta es la forma en que lo percibe Miguel, el niño solitario del relato "El retrato amarillo" (1956), quien se encuentra, sin proponérselo, con la visión de un coito entre dos criados: "Distinguió unas formas blancas, entrelazadas, desnudas. Tardó unos segundos en darse cuenta de que los yacentes eran Maximina y el Absalón, pero no comprendió qué hacían, y fue apoderándose de él un terror de esos que lo sobrecogían y lo dejaban helado, tieso. (...) Sus desnudeces relampagueaban en la gran sombra trémula, como si no fueran un hombre y una mujer sino un ser fabuloso, de nácar fulgente, con tentáculos que sin cesar se retorcían." (15). El personaje, según esta descripción, no alcanza a entender si son uno o más individuos mezclados, si lo que ve es humano o animal, si son una o más especies implicadas. Lo horroroso aquí es la imposibilidad de reconocer y clasificar a este monstruo -y en este sentido, toda racionalidad está suspendida-, del cual el chico destaca sus aspectos más repulsivos: unas "formas blancas y desnudas", comparables a larvas o a tentáculos movedizos.

Mujica Lainez utiliza el término "terror" para caracterizar la sensación del personaje. Sin embargo, de acuerdo a los elementos invoca esta visión, se trata de una emoción más rudimentaria y menos elaborada que el terror. Alberto Manguel (2008) indica que, según la tradición de la novela gótica, el terror y el horror poseen caracteres diferentes. Mientras que éste paraliza y limita al espectador, aquél le provee herramientas, lo fortalece y amplía sus límites; el primero es próximo a lo sublime; el segundo, a lo repulsivo. El personaje de Mujica se siente suspendido y limitado frente a la visión desbordante de los cuerpos copulando. Hay aquí no sólo un cuadro de lo que asusta y a la vez paraliza al observador, sino la imagen que desborda el cuadro del que mira. Miguel siente una cercanía que le causa aversión; hay una sobredimensión de la figura que acerca al observador hacia lo observado y amenaza con contaminarlo y envolverlo con su mácula. En este sentido, si hay algo que motive la sensación de repulsión, según el pensador italiano Mario Perniola (2002), es la vida que desborda la vida, la vida sin forma ni control, la vitalidad de aquello que amenaza con contagiar y corromper a lo que rodea, como aquella que se desprende de la escena de unos cuerpos que escapan a cualquier taxonomía. 
En la mirada de Miguel hay miedo y disgusto, pero a la vez deseo. Jeffrey Cohen (1996) en sus "Siete tesis" sobre la monstruosidad, señala a propósito que el miedo hacia el monstruo es en realidad un deseo. El ser anómalo, en tanto cuerpo - frontera de aquello inexplorado y prohibido, da cuenta de la corporización y el retorno de lo reprimido. El pavor que trae su aparición moviliza el deseo de quien lo observa; su cuerpo terrorífico, horroroso o abyecto irrumpe en el plano de las certezas racionales invocando un aspecto oculto y erotizado. Este argumento de Cohen -de clara raíz psicoanalítica- enlaza miedo y deseo, cultura y represión. El lugar donde se esconde lo monstruoso, en consecuencia, es un intersticio entre el miedo y la atracción, y suscita emociones en quienes observan este límite endeble que separa el temor del deseo ${ }^{3}$.

La mirada de espía se desliza hacia el reflejo de los espejos en la novela Los cisnes (1977). En esta narración, la bailarina clásica Noemí es presa del deseo en el momento en que se encuentra furtivamente con el seductor Efraín en un guardamuebles atiborrado de viejos e inquietantes trastos (nuevamente lo atemorizante es contiguo al erotismo). Al inicio de la escena, los monstruos, amenazantes, parecen rodear a los amantes, encarnándose en el antiguo mobiliario que los circunda: "los atisbaban en derredor, como dragones, como hipogrifos, como basiliscos y harpías, los armarios, las vitrinas, los aparadores." (170). En paralelo a la entrega sexual y al alejamiento de las culpas, la monstruosidad se mezcla con los cuerpos de los amantes en el momento en que los espejos comienzan a exhibir y descubrir su imagen multiplicada, y en este sentido, fuera de toda clasificación: "las distintas lunas, quebradas o enteras, nebulosas o precisas, registraban cada uno de sus gestos, cada uno de sus ímpetus y desmayos (...) de suerte que se hubiera dicho que el desván no albergaba una sola pareja de amantes, sino cuatro, ocho, diez acoplamientos enardecidos los cuales actuaban ciñéndose al mismo y furioso compás." (Ibíd.). Como en "El retrato amarillo", los cuerpos unidos dan lugar a la visión del monstruo constituido por la pérdida de diferencias: la criatura es una bestia fuera de la medida: indeterminada, profusa y lasciva.

En la novela El viaje de los siete demonios (1974), Asmodeo, el demonio de la lujuria, tiene una apariencia confusa (indiferenciada) y grotesca:

3 Marie-Hélène Huet en Monstrous imagination (1993) estudió la relación entre deseo y monstruosidad, ligada no ya a la visión de lo monstruoso (como se trabaja en "El retrato amarillo") sino al efecto de los apetitos de las madres en la gestación y el embarazo sobre sus futuros hijos. 
un cuerpo homínido, orejas de conejo y hocico de cerdo; parece haber atravesado las especies tomando un poco de algunas de ellas. Posee asimismo el poder de cambiar de apariencia, desde esta inicial, hacia otras, más atractivas para los futuros pecadores. "A veces" -comenta el narrador- "se transformaba en una mujer o en un adolescente, desnudos ambos y tan cambiantes que resultaba imposible discernir su sexo." (1974, 21). La monstruosidad de Asmodeo combina la hibridación entre especies con el desvanecimiento de la diferencia sexual. La imagen de las versiones humanas del demonio (la mujer y el adolescente desnudos) pone de manifiesto la imposibilidad de diferencias ya que el que observa a este ambiguo y bello cuerpo no puede fijar la orientación de su deseo: es una atractiva muchacha y un hermoso joven a la vez, llamándolo a abandonar la castidad. Esta modalidad mutante del deseo es la herramienta principal de Asmodeo, quien, para cautivar y lograr que la víctima caiga en la lujuria, cambia de aspecto continuamente, a saber -la lista es tan larga como hilarante-: "una geisha del Japón, un efebo espartano, una hetaira del Corinto, un travesti brasileño, Safo de Lesbos, el amante de Lady Chatterley, una Emperatriz de Bizancio, el Príncipe de los Lirios de Creta, una prostituta de Hamburgo, un paje del Renacimiento, las dos majas de Goya, Lord Alfred Douglas, un hada de Las Mil y Una Noches, un hermafrodita de cualquier parte, don Juan y la Bella Otero." (265). A pesar del atractivo de cada figura del caleidoscópico Asmodeo, el resultado es más bien en una masa confusa, como si fuese un grotesco "humano y voluptuoso pulpo" (ibíd.)4, de modo que la construcción del sexo queda ligada, nuevamente, con la imagen de una bestia que se escapa de las clasificaciones. Se trata de un aspecto, este último, propio de la aparición de las monstruosidades, según lo señala Cohen (1996): presagiar la crisis de los conceptos socioculturales, en este caso, la configuración de las sexualidades desligadas del binarismo frente

4 En este sentido, el texto de Mujica Lainez se acerca al "realismo grotesco" con el que Mijail Bajtín describía imaginarios y prácticas culturales durante la Edad Media y el Renacimiento. Efectivamente, Bajtín (1990) caracteriza al cuerpo "grotesco" no como un físico individualizado sino en un intersticio o "metamorfosis incompleta" (entre la vida y la muerte, entre el cielo y la tierra) que lo hace a la vez profuso, abierto e incompleto. Dicha imagen alegoriza el plano material y corporal ("inferior") propio de las representaciones populares, contrarias a las narraciones dominantes (principalmente las que impartía la Iglesia) del Medioevo europeo. Desde este punto de vista, el cuerpo monstruoso se convierte en una construcción contrahegemónica.

Es necesario destacar, en relación con el plano profano que invoca Bajtin, que para la escritura de El viaje de los siete demonios (como para otras novelas como Bomarzo [1962] y El unicornio [1965]) el escritor argentino consultó varios tratados de demonología y magia, algunos de ellos conservados en la biblioteca de su casa de Cruz Chica (provincia de Córdoba), que este investigador pudo constatar personalmente. 
a la crisis de un heterosexismo dominante ${ }^{5}$. Por otra parte, a diferencia de "El retrato amarillo", Asmodeo guarda un componente novedoso: en sus figuras hay cuerpos deslumbrantes y seductores. Lo que los vuelve inquietantes es la metamorfosis. Cada una de las estampas evanescentes de belleza es el reverso de las transformaciones diabólicas de Asmodeo, haciendo que los cuerpos deseables unificados, indiferenciados y confundidos resulten aborrecibles. Las figuras de la lista sugieren belleza de manera individual; son los cambios los que hacen que el cuerpo se torne tan profuso como repulsivo.

La individualidad de cada figura detenida y racionalizada emana belleza, como la que un observador podría tener frente a una galería de figuras esculpidas. En Mujica Lainez, la escultura truncada refleja un ideal quebrado cuya amputación refleja un límite estrecho entre armonía y monstruosidad. El torturado duque de Bomarzo, por ejemplo, recibe como regalo una antigua figura de Minotauro en la que ambos planos aparecen confundidos. "El torso de atleta" -observa el noble italiano- "sin piernas y sin brazos y con el sexo salvado prodigiosamente, se coronaba con una cabezota cuyo horror no procedía tanto de los rasgos bestiales y del casquete crespo que las ceñía, entre las orejas puntiagudas y rotas, como de la bárbara mutilación que había sufrido en plena cara y que le había arrancado buena parte de ella. El contraste entre la fascinadora voluptuosidad de ese cuerpo armónico y esa testa monstruosa, me espantó en el primer instante" porque "era un símbolo inquietante: la quimera pavorosa, bella y repulsiva." (2007 [1962], 471). Con estas reflexiones, el personaje descubre la ambivalencia estética que guarda la escultura. La monstruosidad corresponde menos a la animalidad del engendro de Creta, prácticamente eliminada, que a la interrupción de la perfección. La mutilación edifica la monstruosidad de la figura pues patentiza dramáticamente la belleza perdida. El duque reconoce su propia anomalía física en la de la estatua; adivina que el regalo que le han otorgado acaso no sea sino una burla por su condición de jorobado y de tullido. El Minotauro, dos veces monstruo, espeja y deforma los miedos

5 Sobre las críticas a las sexualidades binarias, ver los clásicos de Eve Sedgwick, Epistemología del armario (1998) y de Judith Butler El género en disputa (2006).

Respecto de la crisis del heterosexismo dominante, el ensayista Héctor A. Murena señalaba en el artículo "La erótica del espejo" (1959), publicado en un espacio central para la cultura argentina como la revista Sur, la extenuación del deseo en el matrimonio burgués, espacio en el que se había estabilizado un horizonte de expectativas y, por lo tanto, se había agotado el erotismo que se desprende de aquello oculto e irracional. 
de quien observa y se observa en él remarcando aquellos rasgos que lo alejan de un ideal estético.

También en "El retrato amarillo" hay una copia de Venus cercenada, cuya cabeza rota cuelga a un lado, pero además, dada su desnudez, ha sido pudorosamente cubierta con un lienzo. Una noche ese lienzo cae y el protagonista del relato la descubre. "Su desnudez lechosa resaltaba en la negrura, como un ambulante cadáver degollado que se hubiera detenido antes de proseguir su tránsito aterrador por salas y galerías" (1956, 30), repara el narrador. Otra vez lo que causa terror al observador es la palidez, no porque tal blancura se acerque a la sensualidad del cuerpo despojado sino porque se asemeja a la de un cadáver o a una sustancia abyecta que conjura la belleza. Mujica provoca aquí una inversión fortísima: hace que el niño vea un monstruo en Venus, el ideal de la beldad y del erotismo occidental. El descubrimiento del cuerpo desnudo, que podría ser una visión sensual, se metamorfosea en un monstruo. La espantosa desnudez de Venus trae nuevamente la sensación de asco y de deseo con que se presenta esta asociación entre monstruosidad y sexo. Tanto es así, que Miguel, en vez de huir de la estatua, camina hacia ella, la abraza y besa los pechos fríos de la Venus, antes de correr asustado hacia su cuarto.

De esta manera, en la primera de las visiones de Miguel (la del sexo) y en la descripción de Asmodeo, la monstruosidad se esconde en la transformación móvil y contaminante de los cuerpos; en las estatuas, en cambio, lo monstruoso se esconde en la mutilación. Si la estatua es la fijación posesiva de la mirada frente a la inestabilidad del mundo, la estatua quebrada y rota da cuenta del rápido desvanecimiento de la belleza 6 .

Las estatuas de Mujica también son los cuerpos de plena belleza sobre los que se centra el deseo obsesivo. Así, para el duque de Bomarzo, Julia Farnese, con quien se casa pero se ve impedido en la intimidad de la alcoba, se asemeja a una gélida y hermosa estatua que lo empequeñece y le impide el sexo: "Con su cabello suelto y su largo ropaje blanco era una aparición lunar. Brillaban como aguamarinas, bajo las pestañas negras, sus ojos transparentes." (398). Más adelante observa: “Mi mujer seguía

6 Otro ejemplo del desvanecimiento de la belleza puede encontrarse en el cuento "El pasajero" (de la colección El brazalete, 1979). Sentado en el fondo de un colectivo repleto, hay un bello muchacho cuya belleza "iluminó un instante la monotonía de nuestra pesadumbre" (85), pero fantásticamente, según lo va advirtiendo el narrador cada vez que alcanza a verlo entre el abarrotamiento de pasajeros, va envejeciendo velozmente hasta desaparecer. 
siendo, en la soledad nocturna de nuestra cámara, la misma estatua hermosa y sin velos, la misma obsequiosa, sonriente frialdad." (407). La figura inmóvil, en este caso, es esta mujer hermosa pero inaccesible para el muchacho, que a su lado ve su propia anomalía física acrecentada: la monstruosidad se dilata junto a la armonía e impasibilidad de la estatua. Otra de las mujeres que obsesiona al protagonista de Bomarzo durante su pubertad, Adriana dalla Roza, no sólo no lo corresponde, sino que además lo engaña con su paje. Adriana aparece descrita como una esfinge "por momentos tierna y hasta audaz; por momentos despiadada y traidora." (165- 166). Una esfinge puede pensarse como una bestia mítica en parte humana, en parte animal, que puede ser cruel con el que pasa delante de ella, de allí su cercanía con lo enigmático, tanto que podría tener el mismo aspecto que una imperturbable y enorme piedra, ajena al deseo.

El protagonista de la novela que lleva su nombre, Sergio (1976), está configurado bajo una perspectiva semejante. Centro de las miradas apetitosas de los visitantes de un hotel de la sierra cordobesa, su figura adolescente se asemeja a una estatua angélica de rasgos criollos. En el episodio inicial, Sergio camina sonámbulo y desnudo por la cornisa del edificio. Es el blanco de la atención no sólo por la posibilidad de que caiga sino por el encandilamiento que produce su cuerpo, descrito como una "figura morena que parece esculpida por un tallista de las grandes centurias, maestro en San Sebastianes" $(1976,19)$, una imagen que, al pie de las suspicacias, invoca un fetiche de la cultura gay. El derrotero del personaje a lo largo de la narración conservará una condición semejante, eje del deseo obsesivo de los que a su alrededor quieren tenerlo, sin que ninguno de ellos se corresponda y, finalmente, víctima accidental de las balas cruzadas de Ezeiza ${ }^{7}$, que interrumpirán la belleza atormentadora de Sergio, como si la violencia política no fuera sino la réplica de la mutilación en una admirable obra esculpida con maestría ${ }^{8}$.

7 El 20 de junio de 1973, tras 18 años de exilio, Juan D. Perón regresó a la Argentina. Cientos de miles de militantes fueron a recibirlo al aeropuerto internacional de Ezeiza. Antes del arribo del avión, se enfrentaron violentamente distintas facciones del movimiento peronista, murieron varias personas (el número todavía sigue en discusión) y resultaron heridas cientos de ellas. El ex presidente tuvo que aterrizar de urgencia en otro aeropuerto. El episodio es conocido como la "Masacre de Ezeiza".

8 Este argumento es deudor del planteo de Alejandra Laera en su notable prólogo a la antología de Manuel Mujica Lainez Los dominios de la belleza (2005). 


\section{2.- El monstruo aristocrático}

En otras obras de Mujica Lainez, en cambio, las monstruosidades están configuradas como formas de diferenciación social para la aristocracia. Esto es, la anomalía (física, moral, social) y la excentricidad suponen marcas propias del linaje y del señorío. Lo que en otras clases o estamentos sociales es estigma y rechazo, puede ser para las clases altas el signo de diferencia y de libertad. Libertad, justamente, de ser monstruos, de explorar lo prohibido y de encarnar aquello opuesto a una sociedad normativizada. A esa holgura se refería el escritor en la novela Cecil (1975) cuando se refería a los emperadores romanos: "fueron tristes monstruos, incomprensibles para nuestra mentalidad." (144). Para Caracalla y para Heliogábalo disimular no tenía sentido. Eran descendientes de los dioses y jefes de un imperio que desbordaba los horizontes de la Antigüedad, ¿por qué habrían de ser rectos? La medida intermedia, la conducta normativa era un parámetro para las muchedumbres. Sólo los monarcas -y por extensión, la fracción social que los rodeaba- tenían la posibilidad de ser monstruos y de realizar todos los excesos que se les antojaran?.

Bomarzo (1962) puede ser leída, en este sentido, como el descubrimiento y la elección de la monstruosidad como afirmación de la clase. El protagonista de esta novela ambientada en el Renacimiento italiano, Pier Francesco Orsini, llega al mundo con signos físicos de la diferencia: es jorobado y rengo. Esta condición le vale no sólo las burlas y las vejaciones, sino incluso el intento de eliminación por parte de su padre, para quien la existencia de su hijo es una mancha en la estirpe Orsini. Todo cambia cuando Pier Francesco crece y se da cuenta que su anomalía física puede ser el carácter más claro de su casta; y, aun más, que su diferencia puede completarse con la inmoralidad. Así, el niño vejado y débil se convertirá en el duque de Bomarzo, el soberbio, el fratricida, el lascivo, el oscurantista, el extravagante. A su alrededor va reuniéndose una "corte de los milagros" -efectivo anacronismo de Mujica- no conformado por jóvenes o bellos, sino por mutilados de las guerras, viejas meretrices, un nigromante siniestro, asesinos en fuga y espectros. Ellos no merman el abolengo del duque, por el contrario, hacen que la casa

9 Otro fundamento para este punto de vista puede encontrarse en ese "fuera de norma" que Mujica les adjudica a las clases tradicionales, por la razón de que serían ellas mismas las que crean esas reglas. En su ensayo sobre Mariquita Sánchez, el escritor advierte: "no tienen por qué ceñirse a las fórmulas, pues las inventan, ya que las fórmulas existen, precisamente, para quienes no se atreven a hacerlas a un lado." (1982, 54- 55). 
Orsini sea la más distinguida, la diferente, la efectivamente aristocrática. El conjunto de esculturas terribles y extravagantes que ordena erigir en sus jardines tiene el mismo sentido. El gusto esotérico y estrafalario de su proyecto refleja una medida profusa de las propias prácticas y elecciones del duque; el tamaño de las esculturas señala la elección por aquello que está fuera de toda sensatez. Sólo la cercanía de su propia muerte lo hace descubrirse como uno más, no es ya el duque predestinado a la vida sin fin, puesto que muere siendo sólo un "pobre monstruo pequeño, ansioso de amor y de gloria, pobre hombre triste." (2007 [1962], 696).

No es difícil encontrar en las novelas anteriores a Bomarzo rasgos que comparten el duque italiano con los personajes de la alta sociedad porteña. En La casa (1954), por ejemplo, el imaginario de la medida excesiva se concentra en el personaje de Clara, gradualmente voluminosa ella misma, que atiborra en su cuarto los más variados objetos exóticos, primero orientales y luego tallas y figuras cristianas, "hasta que casi fue imposible moverse en ese ámbito colmado por los objetos" (1995 [1954], 40). Piénsese en el contraste entre la desproporción entre esta habitación recargada con la medida equilibrada que parecía gobernar el resto de la residencia de la distinguida calle Florida, centro de sociabilidad de las elites porteñas en las últimas décadas del siglo XIX y las primeras del XX. La profusión puede pensarse asimismo como un gesto de conservación frente a las alteraciones extramuros. Susan Stewart (2007) señala que las miniaturas y el afán coleccionista tienen un efecto de clausura para el observador, de manera tal que le otorga posesión y control sobre ellas. Si las colecciones de estatuillas devotas, muñecas, caretas y fruslerías japonesas que copan la habitación pueden considerarse como dobles ideales del mundo, su exceso parece ser una respuesta exagerada frente a las transformaciones externas percibidas como amenazantes. Los años que recorre la novela de Mujica Lainez van acompañados por profundas alteraciones sociales, culturales y políticas fuera de la vivienda. El monstruoso cuarto de Clara quiere ser, en este sentido, el reverso conservador del cambio social.

En la novela Invitados en 'El Paraíso' (1957) no hay monstruos pero sí los gestos de la extravagancia de la aristocracia. La quinta "El Paraíso" y sus alrededores, un refugio para una familia de clase alta desplazada, es un mundo situado en una frontera entre lo fantástico, lo alienado, lo mágico y lo excesivo. La "corte” aquí está formada, entre otros, por la nictálope señora Tití -cuyos orígenes no son nada nobles, aunque se 
dice hija del señorío portugués- que se levanta a las once de la noche y sale a caminar por sus extensas tierras. Ella "ha cambiado las horas al revés" -comenta su hijo- "como quien da vuelta un guante." (1991 [1957], 27). Miss Lucy Landor, otro de los habitantes de "El Paraíso", es una extraña dama británica obsesionada con ver hadas en cada rincón. Tony (hijo de Tití) y sus ayudantes despliegan desmesurados proyectos cinematográficos que comienza y nunca termina de filmar; uno de ellos, por ejemplo, ambientado en el siglo XVII y que intenta, valga el humor de Mujica, armonizar "la gran tradición barroca italiana del siglo XVII y XVIII con las lecciones del cine expresionista alemán de 1920." (22). Para los visitantes invitados, el pintor Silvano y su ayudante, el espectáculo de libertades extravagantes de "El Paraíso" no puede pertenecer a la realidad, y ni siquiera al mundo de los vivos. Si ésas son sus rutinas, si esos son sus medidas de la vida y de las actividades cotidianas, quienes viven allí no pueden estar con vida, deben ser cadáveres. "Están embalsamados o están muertos." -comenta con temor Silvano-. "Aquí no hay ni una persona viva." (31). No advierte que todas esas rarezas, todos estos lenguajes, cuerpos y hábitos extraños son los signos a destiempo de una clase social que entraba en ocaso.

\section{Conclusiones}

El recorrido por la narrativa de Manuel Mujica Lainez pone de manifiesto tres configuraciones a la vez diferenciadas y enlazadas sobre la monstruosidad que dialoga con la coyuntura de profundos cambios socioculturales de la primera mitad del siglo XX en Argentina. En primer lugar, lo monstruoso se revela en la visión del sexo. Bajo este punto de vista, los cuerpos entrelazados se transforman en una bestia tan fabulosa como repugnante y suscitan en el observador el deseo por aquello que le resulta desconocido. En el caso del relato "El retrato amarillo" (1956), esta construcción es especialmente interesante porque la mirada erotizada se direcciona desde los ojos de un púber de clase acomodada hacia las clases bajas, y en este sentido delata el deseo que produce este otro social. En este y en otros textos de Mujica, la representación de lo monstruoso revela, así, menos un límite infranqueable que una crisis de categorías sociales y culturales: la alteración de la lógica social y la crisis del binarismo sexual, entre otros.

Una segunda construcción de lo monstruoso, contigua a la anterior, es la que se asienta sobre las estatuas. Mujica Lainez las presenta 
mutiladas y degradadas, contradiciendo cualquier idea de belleza o armonía detenida en la piedra. En esas figuras rotas se enfatiza, de este modo, la nostalgia por una belleza perdida irrecuperable, un aspecto que, leído en el contexto de los años 1950 y 1960, puede verse como el desmoronamiento de un imaginario estético paralelo al desplazamiento del patriciado.

Finalmente, Mujica Lainez recurre en sus textos a la idea de monstruosidad moral (metaforizada, en el caso del duque de Bomarzo, en el cuerpo anómalo). En efecto, otorga a algunos de sus personajes aristocráticos la libertad de adoptar prácticas inmorales, de escapar a la línea normativa que rige para el resto de las clases. La monstruosidad es edificante de las fracciones sociales altas, y bajo esta mirada, el escritor enfatizaba un gesto de diferenciación frente a la sociedad y la cultura de masas.

\section{Bibliografía}

Bajtin, Mijaíl. La cultura popular en la Edad Media y en el Renacimiento. El contexto de François Rabelais. Madrid: Alianza, 1990.

Butler, Judith. Deshacer el género. Barcelona: Paidós, 2006.

Cohen, Jeffrey (ed.). Monster theory. Reading culture. Minneapolis: University of Minnesota Press, 1996.

Cruz, Jorge. Genio y figura de Manuel Mujica Lainez. Buenos Aires: Eudeba, 1997.

Gallo, Gastón. “Manuel Mujica Lainez. El amplio gesto de la narración”. En: Saítta, Sylvia (dir.). Historia crítica de la literatura argentina. Tomo IX: El oficio se afirma. Buenos Aires: Emecé, 2004. 483-499.

Gilman, Claudia. Entre la pluma y el fusil. Debates y dilemas del escritor revolucionario en América Latina. Buenos Aires: Siglo XXI, 2003.

James, Daniel (Comp.). Nueva historia argentina. Tomo IX: Violencia, proscripción y autoritarismo (1955-1976). Buenos Aires: Sudamericana, 2007. 
Huet, Marie-Hélène. Monstrous imagination. Harvard University Press, 1993.

Manguel, Alberto. "La novia de Frankenstein". En: Shelley, Mary, Frankenstein o el moderno Prometeo. Buenos Aires: Sudamericana, 2008. 13-73.

Mujica Lainez, Manuel. "El retrato amarillo". Ficción 3 (Buenos Aires, setiembre- octubre de 1956). 11-62.

—_. Cecil. Buenos Aires: Sudamericana, 1975.

___ El viaje de los siete demonios. Buenos Aires: Sudamericana, 1974.

—_. Sergio. Buenos Aires: Sudamericana, 1976.

—_. Los cisnes. Buenos Aires: Sudamericana, 1977.

.. El brazalete y otros cuentos. Buenos Aires: Sudamericana, 1978.

_- Páginas de Manuel Mujica Lainez seleccionadas por el autor. Buenos Aires: Celtia, 1982.

__. Invitados en el Paraíso. Buenos Aires: Planeta, 1992 [1957].

—_. La casa. Buenos Aires: Sudamericana, 1995 [1954].

- L Los dominios de la belleza. Antología de relatos y crónicas. Buenos Aires: Fondo de Cultura Económica, 2005. Selección y prólogo de Alejandra Laera.

—_. El arte de viajar. Antología de crónicas periodísticas (1935-1977). Buenos Aires: Fondo de Cultura Económica, 2007. Prólogo y selección de textos de Alejandra Laera.

__. Bomarzo. Buenos Aires: Sudamericana, 2007 [1962].

Murena, Héctor. “La erótica del espejo”. Sur 256 (enero - febrero de 1959). 18-30.

Perniola, Mario. El arte y su sombra. Madrid: Cátedra, 2002.

Sedgwick, Eve K.. Epistemología del armario. Barcelona: Ediciones de la Tempestad, 1998.

Stewart, Susan. On longing. Narratives of the miniature, the gigantic, the souvenir, the collection. Nueva York: Duke University Press, 2007. 
Terán, Oscar. Nuestros años sesentas. La formación de la nueva izquierda intelectual en Argentina, 1956-1966. Buenos Aires: Puntosur, 1991.

Torre, Juan C. (Comp.) Nueva historia argentina. Tomo VIII: Los años peronistas (1943- 1955). Barcelona: Sudamericana, 2002.

Villordo, Oscar Hermes. Manucho. Una vida de Mujica Lainez. Buenos Aires: Planeta, 1991. 